\title{
Desenvolvimento de Sistemas Complexos aplicáveis aos Sistemas Inteligentes de Transporte (ITS) - O caso SINIAV
}

\author{
Alexandre Rojas \\ IME/UERJ \\ rojas@ime.uerj.br
}

\author{
Antonio Carlos de A. Ritto \\ IME/UERJ \\ ritto@ime.uerj.br
}

\author{
Paulo Cezar M. Ribeiro \\ COPPE/UFRJ \\ pribeiro@coppe.ufrj.br
}

\begin{abstract}
Resumo
$O$ desafio de integrar várias aplicações computacionais é comum em engenharia Estes desafios também enfrentam os engenheiros de transporte. Sistemas de controle, tais como sistemas de controle de tráfego e sistemas de gestão de rodovias, desempenham um papel central nos Sistemas Inteligentes de Transporte (ITS). Embora estes sistemas forneçam o benefício individualmente, os seus potenciais não são inteiramente alcançados até que sejam integrados regionalmente. A integração regional permite aos sistemas compartilhar informações, possibilitando um melhor gerenciamento de todo o sistema de transporte regional. Este artigo aborda estes aspectos da computação e propõe algumas tecnologias para o Sistema Nacional de Identificação Automática de Veículos - SINIAV - atuar como elemento de desenvolvimento regional e de integração nacional na Gestão e Planejamento do Tráfego.
\end{abstract}

\section{Abstract}

The challenge of integrating multiple computer applications and systems is common in engineering. This challenge also faces transportation engineers. Control systems, such as traffic signal systems and freeway management systems, play a central role in the intelligent transportation systems (ITS) program. While such systems provide benefit individually, their full benefit is not achieved until they are integrated regionally. Regional integration enables the systems to share information, allowing officials to manage the entire regional transportation system. Planning, designing, and developing integrated systems call for developers to make computing decisions. These papers addresses these computing aspects and propose some technologies for the SINIAV be used as a basis to regional planning and managing of traffic.

\section{Introdução}

Os sistemas de transportes têm possibilitado a locomoção de pessoas e bens de forma rápida e eficiente. Entretanto, as facilidades e o status incorporados ao transporte individual e os baixos níveis de serviço oferecidos pelo transporte público foram alguns dos fatores que contribuíram para um aumento substancial da frota nos grandes centros urbanos prejudicando a fluidez do tráfego e gerando congestionamentos cada vez maiores em extensão e tempo.

Embora não exista uma única solução para estes problemas, os Sistemas Inteligentes de Transporte (ITS Intelligent Transportation Systems), oriundos da união da aplicação massiva da telemática e de ferramentas de gerenciamento e controle, apresentam-se como um novo instrumento a ser utilizado de forma exclusiva ou em colaboração com as técnicas tradicionais de transporte e tráfego.

Este trabalho procura identificar as tecnologias emergentes que possam dotar o Sistema Nacional de Identificação Automática de Veículos - SINIAV - com funcionalidades para a Gestão e Planejamento do Tráfego.

O SINIAV, em sua concepção legal, objetiva capacitar os órgãos executivos de trânsito com instrumentos modernos e interoperáveis para planejamento, fiscalização e gestão do trânsito e da frota de veículos, baseado em tecnologia de identificação por rádio-freqüência, e que em uma visão mais ampla pode ser considerado, por suas funcionalidades, como integrante de um Sistema Inteligente de Transporte ITS.

\section{O Problema da Pesquisa}

O desenvolvimento de Sistemas de Informação voltados aos Sistemas Inteligentes de Transporte - ITS vem representando um dos mais ambiciosos projetos da engenharia de transporte. Todavia, que, a exemplo dos países onde a implantação do ITS já se encontra em um estágio mais avançado, a integração dos vários sistemas está provando ser uma difícil empreitada.

No caso brasileiro, a prática demonstra que os Órgãos Executivos de Trânsito, independente da esfera administrativa, têm anos de experiência na implantação da infra-estrutura física, porém o desenvolvimento dos sistemas computacionais ligados à gestão e controle do transporte é realizado de forma autônoma sem a preocupação de se construir uma integração regional.

Com isso, é provável que se mantenha uma determinada realidade orçamentária e uma orientação política local, mas em contrapartida comprometem-se os ganhos globais na melhoria do transporte que podem advir da integração.

Dois projetos específicos, um nos Estados Unidos da América patrocinado pela American Association of State Highway and Transportation Officials AASHTO e outro na União Européia denominado Cooperative Vehicle- 
Infrastructure System-CVIS vêm trabalhando de forma a estabelecer um padrão no desenvolvimento das aplicações computacionais ligadas ao ITS.

Comprometido com os problemas e características locais, ao lado de uma integração nacional que extraia vantagens da complementariedades entre diversas regiões, o objetivo deste trabalho é identificar as principais tecnologias que possam ser utilizadas pelo SINIAV de forma a dotá-lo de instrumentos adequados ao planejamento e gestão do transporte servindo de base para uma futura arquitetura de ITS brasileira.

\section{Arquitetura Básica de um Sistema ITS}

\subsection{Conceitos Gerais}

Um sistema [1] é definido pela sua estrutura em termos de objetivos, componentes, conexões e limitações. A arquitetura dos sistemas não define os detalhes de todos os componentes, como hardware ou software, nem define protocolos específicos de comunicação entre os elementos do sistema. Além disso, o tamanho e a complexidade de uma arquitetura são dependentes do tamanho e da complexidade do sistema. Em alguns casos, como um pequeno sistema de sinalização de tráfego, a maioria dos elementos da arquitetura são peças de equipamento ou componentes de software. Por outro lado, elementos de arquiteturas em grande escala, como a integração de países como o Mercosul, tendem a ter os próprios sistemas de trânsito. Diante da diversidade e heterogeneidade de elementos envolvidos, um ITS é, certamente, um sistema complexo.

A noção de um Sistema Complexo (complex system of systems SoS) é um conceito abstrato, muitas vezes sem uma manifestação física que representa a totalidade cognitiva, na qual um conjunto de sistemas operado em conjunto e em harmonia uns com os outros sistemas proporciona maior funcionalidade do que a soma das partes. Assim, um SoS aumenta a complexidade dos programas introduzindo a necessidade de gerir vários pedaços, cada um representando um sistema complexo com características próprias.[2].

O paradigma da complexidade surge das novas descobertas nos sistemas naturais - autopoiesis, teoria do caos, física quântica, estruturas dissipativas etc - que incluem a incerteza, a auto-produção e a complexidade[3]:

- Incerteza e indeterminação - não podemos ter controle sobre o futuro - comportamento não mecânico e não linear;

- Auto-produção e auto-organização - geração e produção da própria organização;

- Complexidade - relações complexas, recíprocas e indeterminadas.

\section{O Sistema Nacional de Identificação Automática de Veículos - SINIAV}

O SINIAV foi criado pela Resolução 212 do CONATRAN de 13 de novembro de 2006, [4] e modificado pela Portaria 85 de 5 de novembro de 2009 quando foram estabelecidos novos prazos e normas técnicas a serem atendidas para sua implantação em todo o país. Atualmente o projeto encontra-se em fase de avaliação pelos órgãos técnicos e pela sociedade quanto a tecnologia a ser adotada. O prazo de implantação do sistema pelos diversos Detrans deve ter inicio em 30 de junho de 2011 e estar concluído em 30 de junho de 2014.

Trata-se de um sistema nacional de identificação veicular com a utilização de tecnologia por rádio freqüência usualmente denominada de RFID (RadioFrequency IDentification) contendo dados do veículo gravados em uma tag ou tansponder, uma antena e um sistema associado capaz de ler e validar os dados recebidos. O projeto do Sistema SINIAV admite além das tags passivas, que respondem ao sinal enviado pela base transmissora, as tags semi-passivas e as ativas, dotadas de bateria, que lhes permite enviar o próprio sinal. Os fabricantes de veículos poderão optar por uma das tecnologias disponíveis desde que obedeçam aos padrões estabelecidos nas Normas Governamentais.

Os objetivos do SINIAV englobam:

- Acompanhamento do Ciclo de Vida do Veículo;

- Fiscalização Urbana e Rodoviária;

- Gestão de Trânsito;

- Recuperação de Veículos;

- Gestão de Meios de Pagamento e Seguro de Veículos;

- Gestão do Transporte de Cargas e Logística.

Para um melhor entendimento do sistema apresentamos a visão geral da topologia .

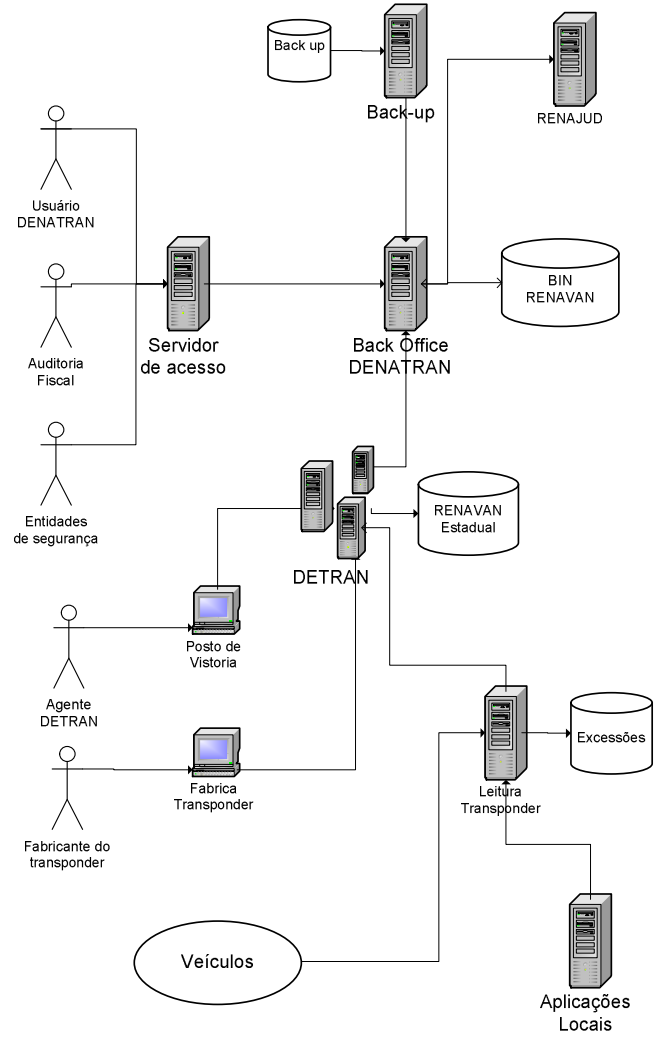

Figura 1 - Visão Geral da Topologia do SINIAV 
Esta topologia foi apresentada pelo DENATRAN em palestra de outubro de 2009, [5] e não é um projeto de sistema nem é um conceito de design. O que ela faz é definir o quadro em torno da quais várias abordagens de design podem ser desenvolvidas, cada uma adaptada para atender às necessidades específicas do órgão, mantendo os benefícios de uma arquitetura comum.

Para enfatizar os aspectos de segurança do sistema ressalta o DENATRAN:

"Sendo um sistema nacional, o risco associado a soluções tecnológicas que não prevejam robustez para vários anos à frente é significativo, especialmente em vista do investimento associado em infra-estrutura de leitores, placas de identificação veicular eletrônica e sistemas de gestão de dados que estará sendo realizado.”[5]

\section{Tecnologias para desenvolvimento e integração do SINIAV}

A topologia do SINIAV mostrada na Figura 1 nos sugere um elenco de tecnologias que permita desenvolver e integrar as aplicações complexas espalhadas geograficamente e de objetivos distintos.

\subsection{Ontologia}

A primeira das tecnologias envolvidas decorre da dificuldade dos diversos atores envolvidos em estabelecer uma linguagem comum que permita o entendimento de um conjunto de conceitos dentro de um domínio e os relacionamentos entre estes.

Ensina-nos a Professora Neide Santos et all[6] que as ontologias são definidas como a especificação formal de um vocabulário de conceitos e axiomas relacionando-os. Ontologias permitem-nos expressar as relações complexas dentro de uma área de domínio. Estabelece relações entre conceitos que nos permitem compreender o conceito não apenas por suas propriedades, mas por sua presença em relação a outros conceitos dentro da ontologia.

Ontologias são úteis para apoiar a especificação e implementação de qualquer sistema de computação complexo. Uma ontologia pode ser desenvolvida para vários fins, mas, de uma forma geral, os seguintes propósitos são atingidos:

- Ajuda as pessoas a compreender melhor certa área de conhecimento: no desenvolvimento de uma ontologia, as pessoas envolvidas no processo se vêem diante de um desafio: explicar seu entendimento sobre o domínio em questão, o que as faz refletir e melhorar sua compreensão sobre esse domínio;

- Promove o consenso sobre uma área de conhecimento, geralmente diferentes especialistas têm entendimento diferenciado sobre os conceitos envolvidos. Ao se construir uma ontologia, essas diferenças são explicitadas e busca-se um consenso sobre seu significado e sua importância;

- Permite que uma pessoa que deseje aprender mais sobre uma área de conhecimento não precise se reportar sempre a um especialista. Ela pode estudar a ontologia e aprender sobre o domínio em questão, absorvendo um conhecimento geral e de consenso.

\subsection{Grids Computacionais}

A computação em grade (Grid Computing) consiste de muitos conceitos e pode ser percebida de várias maneiras, mas na sua essência, ela prevê uma computação distribuída fazendo uso de recursos virtuais. O sistema permite a virtualização de computação distribuída possibilitando que dados e demais recursos como capacidade de processamento, largura de banda de rede e de armazenamento possam criar uma única imagem do sistema. Um usuário da rede vê o sistema como um grande computador único. Em seu núcleo, a computação em grade se baseia um conjunto aberto de normas e protocolos como o Open Grid Services Architecture (OGSA), que permite a comunicação através tecnologias heterogêneas ou ambientes geográficos distantes. Usando Grid computing organizações podem otimizar computação, bem como compartilhar os recursos de dados, otimizá-los quando há grande capacidade de cargas de trabalho, disponibilizá-los através de redes e permitir colaboração e muito mais.[7]

Os atrativos desta idéia incluem a possibilidade de fornecimento de qualquer serviço computacional sob demanda, o qual pode ser composto dinamicamente por outros serviços e agregar recursos localizados em várias instituições distintas e geograficamente dispersos. Além disso, os recursos podem ser alocados em uma quantidade enorme e por um custo muito menor do que alternativas tradicionais baseadas em supercomputadores paralelos.

Um aspecto importante, que deve ser considerado, é o fato de que mesmo havendo a convergência entre tecnologias para o alto desempenho computacional, existe uma leve diferença entre Grids que fornecem e que não fornecem um serviço de execução remota.

Esse serviço é fundamental para aplicações de ITS que necessitam de um processamento de alto desempenho, uma vez que demandam a execução de aplicações paralelas no Grid.

De modo geral, os Grids são mais distribuídos, diversos e complexos que outras plataformas. Alguns aspectos que evidenciam esta distribuição, diversidade e complexidade são:

- Heterogeneidade: Os componentes que formam a infraestrutura tendem a ser extremamente heterogêneos. Ou seja, é importante ter em mente que qualquer solução para Grids Computacionais deverá lidar com recursos de várias gerações, softwares de várias versões, instrumentos e serviços dos mais variados tipos;

- Alta dispersão geográfica: Essa característica se refere a escala que um Grid pode atingir. Nesse sentido, Grids podem ter escala global, agregando serviços 
localizados em várias partes do país;

- Compartilhamento: Em um Grid os recursos são compartilhados todo o tempo e, em contraste com soluções space-shared, um Grid não pode ser dedicado a uma aplicação de forma exclusiva por um determinado período de tempo. Isso tem impacto no desenvolvimento de aplicações que executam sobre a infraestrutura de um Grid Computacional;

- Múltiplos domínios administrativos: Grids congregam recursos de várias instituições. Sendo assim, além da heterogeneidade mencionada anteriormente, é possível também a existência de várias políticas de acesso e uso dos serviços, de acordo com as diretrizes de cada domínio que faz parte do Grid;

- Controle distribuído: Tipicamente não há uma única entidade que tenha poder sobre todo o Grid. Isso é um reflexo da dispersão dos componentes do Grid, pois cada instituição pode implementar sua política em seus recursos específicos, mas não interfere diretamente na implementação de políticas no acesso aos serviços de outras instituições participantes.

\subsection{Web Semântica ou Inteligente}

A Web Semântica ou Inteligente, vem se apresentando como solução para ordenar o caos informacional existente na web.

Segundo Berners-Lee et alii[8], a web semântica será uma evolução da web atual porém apresentará estrutura que possibilitará a compreensão e o gerenciamento dos conteúdos armazenados na web independente da forma em que estes se apresentem, seja texto, som, imagem e gráficos à partir da valoração semântica desses conteúdos, e através de agentes que serão programas coletores de conteúdo advindos de fontes diversas capazes de processar as informações e permutar resultados com outros programas

A web semântica, a exemplo da web atual, será tão descentralizada quanto possível e deverá manter a responsabilidade exigida por esta descentralização, procurando alcançar o ideal de consistência de interconexões, porém permitindo seu crescimento exponencial.

Basicamente, a web semântica se compõe de três elementos: (a) Representação do conhecimento (Knowledge representation); (b) Ontologias (Ontologies) e (c) Agentes (Agents).[9]

(a) Representação do conhecimento

A web semântica possui uma estrutura integrando o conteúdo de páginas web criando um ambiente onde programas através de agentes ou agentes inteligentes poderão percorrer vários sites e assim executar tarefas sofisticadas para os usuários.

(b) Ontologias

A Ontologia, na web semântica, estabelece uma ligação terminológica entre membros de uma comunidade podendo ser estes membros, agentes humanos ou máquinas.

(c) Agentes
A função dos programas agentes ou agentes inteligentes é coletar conteúdos na web a partir de fontes diversas, processar a informação e permutar os resultados com outros programas através de linguagem que expressa inferências lógicas resultantes do uso de regras e informação como aquelas especificadas pelas ontologias. O principio está, não no entendimento, pela máquina, daquilo que está escrito e sim no reconhecimento de provas escritas na linguagem estabelecida pela ontologia, onde os programas-agente, pela inferência lógica, retornam respostas ao que foi requerido, ou agente e consumidor podem alcançar entendimento compartilhado permutando as ontologias, que oferecem o vocabulário necessário para a discussão.

\subsection{O Software Livre como instrumento de desenvolvimento de Sistemas relacionados ao ITS}

A quarta tecnologia proposta é o desenvolvimento das aplicações computacionais para o ITS utilizando Software Livre.

O Software Livre tem em sua essência alguns componentes estruturais, entre eles:

$>$ o uso de padrões abertos,

$>$ o licenciamento livre dos softwares,

$>$ a formação de comunidades, em especial de usuários e desenvolvedores.

O uso de padrões abertos se concretiza nos padrões de interoperabilidade do governo federal, e-Ping. O ePing é um instrumento normativo que se encontra em sua $3^{\text {a }}$ versão e tem como principal característica definir os padrões de interoperabilidade das tecnologias da informação e comunicação adotados pelo governo.

No caso de desenvolvimento de sistemas ligados ao SINIAV o uso de Software Livre além de representar o atendimento a uma política governamental, evita que uma tecnologia proprietária possa levar a dependência e/ou o atrelamento das pessoas ao produto/produtor, o que certamente não é conveniente para o desenvolvimento de Sistemas ligados ao ITS.

Este aspecto é destacado na política Norte Americana através do National ITS Architeture em sua versão 6.1 ( atualizada em 01 de julho de 2009) que preconiza no item 9.do item 1.2 Architeture Strategies and Principles

"Deve-se manter uma arquitetura aberta imparcial com qualquer produto em particular. Todos os sub sistemas da arquitetura poderão interagir com uma gama ilimitada de hardwre e serviços.[10]

\subsection{Framework voltados ao desenvolvimento de aplicações ITS}

A quinta e última tecnologia proposta é a utilização de um framework maduro em termos de 
desenvolvimento e, para tanto, é possível visualizar o emprego do ITS National Architerture ou do FOAM (Framework for Ontology Alignment and Mapping) ambos aplicáveis ao SINIAV.

Neste trabalho será apresentado o FOAM.

O objetivo do FOAM é criar um ambiente de execução aberto, no qual aplicações voltadas ao ITS possam ser desenvolvidas, entregues executadas e mantidas, durante o ciclo de vida dos veículos e da infraestrutura da estrada/cidades [11].

Criada inicialmente para o Projeto CVIS da União Européia, este Framework já se encontra estabilizado quanto à ocorrência de erros e poderá ser utilizado em outras localidades, permitindo que os Engenheiros de Software possam desenvolver aplicações computacionais confiáveis.

O FOAM irá definir uma arquitetura que conecta os sistemas de bordo, infra-estrutura rodoviária e infraestrutura de back-end que é necessária para a co-gestão dos Sistemas Inteligentes de Transportes.

Em outras palavras, o objetivo é produzir uma arquitetura e especificação de forma que a execução seja independente, ou seja, permitir que diferentes implementações de cliente e várias tecnologias de backend server possam convergir e interagir. Para o ambiente de execução de referência, o FOAM poderá criar uma ligação com tecnologias específicas, e estabelecer um sistema totalmente funcional.

Em termos de plataforma de desenvolvimento, o principal candidato para um cliente de ligação é Java / OSGi executando sobre plataforma do sistema operacional Unix, o mais provável NetBSD.

As características principais do Framework FOAM são:

- Possibilidade de intercâmbio e atualização de componentes de aplicações de serviço em qualquer domínio da arquitetura CVIS (mas não limitado ao mesmo);

- Produto com independência de fornecedor de componentes de middleware;

- Requisitos padronizados para elementos estruturais (por exemplo, formatos de intercâmbio de dados, especificações do protocolo, meio ambiente e de API run-time);

- Projeto de comunicação segura em sistemas distribuídos, incluindo faturamento, a autenticação de dados de usuários e autorização de usuários;

- Reutilização de componentes e acesso generalizado aos recursos.

\section{Considerações Finais}

Os exames das Resoluções emanadas pelo CONATRAN e DENATRAN revelam o foco principal na identificação dos veículos voltados ao roubo/ recuperação de veículos e pagamento de tributos. De forma acessória, trata a gestão e planejamento do transporte delegando aos Órgãos Executivos locais o desenvolvimento das aplicações computacionais sem, contudo, tratar de uma integração nacional.

O levantamento das informações obtidas pelo SINIAV, juntamente com as tecnologias de engenharia de software disponíveis, possibilita um desenvolvimento integrado com ganhos significativos para a sociedade.

As tecnologias avaliadas neste trabalho nos possibilitam concluir que:

- O uso de Software Livre para o desenvolvimento das aplicações ligadas ao SINIAV ou ao ITS Nacional possibilitará uma maior transparência nos sistemas;

- O uso de tecnologias ligadas a Ontologia, Grid Computacional e Web Semântica possibilitarão às diversas aplicações a integração nacional desejada;

- O uso de plataformas estáveis e de reconhecida capacidade poderá fornecer a segurança necessária aos sistemas e dados.

Como trabalho futuro é recomendado uma participação junto aos Grupos de Trabalho promovidos pelos Órgãos Reguladores voltados ao desenvolvimento integrado do planejamento e gestão do transporte utilizando os dados obtidos pelo SINIAV.

\section{Referências}

[1]Bertallanfy, L. Teoria geral dos sistemas. Petrópolis: Vozes, 1975.

[2]Stephen B Jr., Steven C, Behm, Evaluating the Software Design of a Complex System of Systems -2010 - Technical Report - disponível em http://www.sei.cmu.edu/reports/09tr023.pdf acesso em 11/02/2010

[3]Ritto, A,C,A- Organizações Caórdicas - $1^{\mathrm{a}}$. Edição - LTC Rio de Janeiro, 2005.

[4]Resolução 212 CONATRAN obtido em www.denatran.gov.br/download/Resolucoes/RE SOLUCAO_212.rtf, 2006, visitado em 29/11/2009

[5]Resumo Executivo dos Requisitos Técnicos http://www.denatran.gov.br/ultimas/20091103_s iniav.htm,2009, visitado em 28/11/2009.

[6]Aparício, A.S.; Farias O L. M; Santos Neide Applying Ontologies in the Integration of Heterogeneous Relational Databases. Australasian Ontology Workshop (AOW 2005), Sydney. Conferences in Research and Practice in Information Technology (CRPIT), Vol. 58. ,2005, T. Meyer and M.Orgun, Ed.

[7]Bali V Singh Rathore R, Sirohi A. Verma P.k Information Technology Architectures for Grid Computing and Applications apresentado no Fourth International Multi-Conference on Computing in the Global Information Technology, August 23-29, 2009 Cannes/La Bocca, French Riviera, France. 2009

[8]Berners-Lee, T.; Hendler, J. e Lassila, O.. The Semantic Web disponível em http://www.sciam.com/article.cfm?articleID $=00$ 048144-10D2-1C70- 
84A9809EC588EF21\&catID=2, 2001, visitado em 29/11/2009.

[9]W3C Semantic Web Activity disponivel em http://www.w3.org/2001/sw/, 2001, visitado em 26/11/2009.

[10]National ITS Architeture Documents Versão 6.1 atualizada em 1/7/2009 disponível em http://www.iteris.com/itsarch/html/menu/ documents.htm, 2009, acesso em 11/02/2010.

[11]CVIS Projetct disponível em http://www.cvisproject.org/en/cvis_subprojects/t echnology/foam/,2007, visitado em 27/11/2009. 